\title{
PSEUDO-OPEN MAPPINGS FROM TOPOLOGICAL SUMS
}

\author{
DENNIS K. BURKE
}

\begin{abstract}
Assume $S$ is the free topological sum of spaces $X_{1}$ and $X_{2}$, on the same set $X$, and $\phi: S \rightarrow X$ is the canonical quotient map. The main result states that $X$ is developable (resp. quasi-developable) if $X_{1}$ and $X_{2}$ are regular developable (resp. quasi-developable) spaces and $\phi$ is pseudo-open. Examples are given to show that certain topological properties are not preserved under such a construction.
\end{abstract}

1. Introduction. Suppose $\Lambda$ is a finite family of topologies on the same set $X$. The family $\Lambda$ is said to be compatible [A] if for every $A \subseteq X$,

$$
\bar{A}_{\cap \Lambda}=\cup\left\{\bar{A}_{\tau}: \tau \in \Lambda\right\} \text {. }
$$

If $S$ is the free topological sum of the spaces $(X, \tau), \tau \in \Lambda$, and $\phi: S \rightarrow X$ is the canonical mapping, the compatibility condition on $\Lambda$ can be stated in terms of pseudo-openness of the mapping $\phi$ as follows.

1.1 Proposition [A]. A finite family $\Lambda$ of topologies on $X$ is compatible if and only if the canonical map $\phi$ from the free topological sum $S$ onto $(X, \cap \Lambda)$ is pseudo-open.

In any case, it is easy to see that the intersection topology $\cap \Lambda$ on $X$ agrees with the quotient topology on $X$ induced by the mapping $\phi: S \rightarrow X$. This gives a slightly different version of Proposition 1.1.

1.2 Proposition. If $\Lambda$ is a finite family of topologies on $X$ then $\Lambda$ is a compatible family if and only if the canonical quotient mapping $\phi: S \rightarrow X$ is pseudo-open.

If each $(X, \tau), \tau \in \Lambda$, has a certain topological property it is natural to ask whether $(X, \cap \Lambda)$ has the same topological property (especially when $\Lambda$ is compatible). It is clear from Proposition 1.2 that such questions can be studied within the framework of mappings. In this note we answer three questions of Arhangel'skii [A] about the intersection of compatible topologies, with the main result saying that the intersection of two regular developable compatible topologies on $X$ is a developable topology. We show this is not necessarily true if one of the topologies is only Hausdorff and give other

Received by the editors March 27, 1978 and, in revised form, May 18, 1978.

AMS (MOS) subject classifications (1970). Primary 54B15; Secondary 54C10, 54E30.

Key words and phrases. Compatible family of topologies, pseudo-open map, quasi-developable, developable, $\sigma$-disjoint base, subparacompact. 
examples showing that certain topological properties are not preserved under the intersection of two compatible topologies.

In the remainder of this paper $X_{1}$ and $X_{2}$ represent topological spaces on the same set $X$ and $S$ is the free topological sum of $X_{1}$ and $X_{2}$. The mapping $\phi: S \rightarrow X$ is the natural canonical mapping and when $X$ is used in this fashion (as the range of $\phi$ ) we assume $X$ has the quotient topology induced by $\phi$. We will not usually distinguish between the sets $X_{1}, X_{2}$ and their disjoint copies used in constructing $S$. This should cause no confusion, but if necessary it may be helpful to use the notation: $X_{1}=X \times\{1\}, X_{2}=X \times\{2\}$, and $S=X_{1} \cup X_{2}$. All topological spaces considered are at least $T_{1}$, and $N$ denotes the set of natural numbers.

2. Intersection of developable compatible topologies. Recall that a space $X$ is quasi-developable if there is a sequence $\left\{\mathcal{G}_{n}\right\}_{1}^{\infty}$ of families of open subsets of $X$ such that whenever $x \in U \subset X$, where $U$ is open in $X$, there is some $n \in N$ such that $x \in \operatorname{St}\left(x, \mathcal{G}_{n}\right) \subset U . X$ is developable if and only if $X$ is quasi-developable and perfect (i.e., all closed subsets are $G_{\delta}$ sets) [Be]. Bennett and Lutzer have shown [BL, proof of Theorem 8] that $X$ is quasi-developable if and only if there is a quasi-development $\left\{\mathcal{G}_{n}\right\}_{1}^{\infty}$ for $X$ such that whenever $x \in U$ and $U$ is open, there is some $n$ such that $x \in \operatorname{St}\left(x, \mathcal{G}_{n}\right) \subset U$ and $\operatorname{ord}\left(x, \mathcal{G}_{n}\right)=1$ (where $\operatorname{ord}\left(x, \mathcal{G}_{n}\right)$ denotes the number of elements of $\mathcal{G}_{n}$ which contain $x)$. For each $G \in \mathcal{G}_{n}$, let $F(G)=\left\{x \in G: \operatorname{ord}\left(x, \mathcal{G}_{n}\right)=1\right\}$ and let $\mathcal{P}_{n}$ be the collection of all pairs $(F(G), G)$, for $G \in \mathcal{G}_{n}$. If $\mathscr{P}=$ $\cup{ }_{n=1}^{\infty} \mathscr{P}_{n}$ we have provided part of the construction necessary for the proof of the next result. The rest of the proof will be left to the reader.

2.1 Proposition. A space $X$ is quasi-developable if and only if there is a family $\mathcal{P}=\cup_{n=1}^{\infty} \mathscr{P}_{n}$ of pairs of subsets of $X$ satisfying:

(a) If $n \in N$ and $P=\left(P^{\prime}, P^{\prime \prime}\right) \in \mathscr{P}_{n}$ then $P^{\prime} \subset P^{\prime \prime}, P^{\prime \prime}$ is open in $X$, and $P^{\prime \prime} \cap P_{1}^{\prime}=\varnothing$ for any $\left(P_{1}^{\prime}, P_{1}^{\prime \prime}\right) \in \mathscr{P}_{n},\left(P_{1}^{\prime}, P_{1}^{\prime \prime}\right) \neq\left(P^{\prime}, P^{\prime \prime}\right)$.

(b) If $x \in U \subset X$, where $U$ is open in $X$, there is some $P=\left(P^{\prime}, P^{\prime \prime}\right) \in \mathscr{P}$ such that $x \in P^{\prime} \subset P^{\prime \prime} \subset U$.

A collection $\mathscr{P}$, of pairs of subsets satisfying (b) of the above proposition, is sometimes called a pair-network for $X$. We will continue to use the notation suggested in Proposition 2.1 where if $P \in \mathcal{P}$ then $P^{\prime}$ denotes the first element of the pair and $P^{\prime \prime}$ denotes the second element.

2.2 TheOREM. Suppose $X_{1}$ and $X_{2}$ are regular quasi-developable spaces on the same set $X$ and $S$ is the free topological sum of $X_{1}$ and $X_{2}$. If the canonical quotient map $\phi: S \rightarrow X$ is pseudo-open, then $X$ is quasi-developable.

Proof. Suppose $\mathcal{G}=\cup_{n=1}^{\infty} \mathcal{G}_{n}$ and $\mathcal{H}=\cup_{n=1}^{\infty} \mathcal{H}_{n}$ are pair-networks for $X_{1}, X_{2}$ respectively, where, with the obvious changes, $\mathcal{G}$ and $\mathcal{H}$ satisfy (a) and (b) of Proposition 2.1. For fixed $n, k \in N$, define

$$
\mathscr{B}(n)=\left\{\text { int } \phi\left(G^{\prime \prime}\right): G \in \mathcal{G}_{n}\right\}, \quad \mathscr{D}(k)=\left\{\text { int } \phi\left(H^{\prime \prime}\right): H \in \mathcal{H}_{k}\right\} .
$$


For $G \in \mathcal{G}_{n}, H \in \mathcal{H}_{k}$, let

$$
F(G, H)=\phi\left(G^{\prime}\right) \cap \phi\left(H^{\prime}\right)-[(\cup \Re(n)) \cup(\cup \mathscr{D}(k))]
$$

and

$$
\mathscr{F}(n, k)=\left\{F(G, H): G \in \mathcal{G}_{n}, H \in \mathcal{H}_{k}\right\} .
$$

Now we show $\mathscr{F}(h, k)$ is a relatively discrete collection-that is, each member of $\mathscr{F}(n, k)$ is contained in an open set which does not intersect any other member of $\mathscr{F}(n, k)$. To see this, pick $F(G, H) \in \mathscr{F}(n, k)$ with $F(G, H)$ $\neq \varnothing$. Note that $F(G, H) \subset$ int $\phi\left(G^{\prime \prime} \cup H^{\prime \prime}\right)$ and int $\phi\left(G^{\prime \prime} \cup H^{\prime \prime}\right) \cap$ $F\left(G_{1}, H_{1}\right)=\varnothing$ if $G_{1} \in \mathcal{G}_{n}, H_{1} \in \mathcal{H}_{k}$ with $G_{1} \neq G$ and $H_{1} \neq H$. Because of this it suffices to show that

$$
F(G, H) \cap \operatorname{cl}\left(\cup\left\{F\left(G, H_{1}\right): H_{1} \in \mathcal{H}_{k}, H_{1} \neq H\right\}\right)=\varnothing
$$

and

$$
F(G, H) \cap \operatorname{cl}\left(\cup\left\{F\left(G_{1}, H\right): G_{1} \in \mathcal{G}_{n}, G_{1} \neq G\right\}\right)=\varnothing .
$$

Suppose $z \in F(G, H) \cap \operatorname{cl}\left(\cup\left\{F\left(G, H_{1}\right): H_{1} \in \mathcal{H}_{k}, H_{1} \neq H\right\}\right)$. Let $z_{1}, z_{2}$ be the preimages of $z$ in $X_{1}, X_{2}$ respectively. Now $z_{2} \in H^{\prime}$, so there is an $X_{2}$ neighborhood $U_{2}$ of $z_{2}$ such that $\bar{U}_{2} \cap H_{1}^{\prime}=\varnothing$ (closure in $X_{2}$ ) for every $H_{1} \in \mathcal{H}_{k}, H_{1} \neq H$ (use regularity and the fact that $H^{\prime \prime} \cap H_{1}^{\prime}=\varnothing$, if $H \neq$ $\left.H_{1}\right)$. Clearly $\phi^{-1}(z)=\left\{z_{1}, z_{2}\right\} \subset G^{\prime \prime} \cup U_{2}$; since $G^{\prime \prime} \cup U_{2}$ is open in $S$ and $\phi$ is a pseudo-open map we have $z \in$ int $\phi\left(G^{\prime \prime} \cup U_{2}\right)$. There exists $y \in$ (int $\left.\phi\left(G^{\prime \prime} \cup U_{2}\right)\right) \cap F\left(G, H_{1}\right)$ for some $H_{1} \in \mathcal{H}_{k}, H_{1} \neq H$. Let $y_{1}, y_{2}$ be the preimages of $y$ in $X_{1}, X_{2}$ respectively; then $y_{2} \in H_{1}^{\prime}$ and $\bar{U}_{2} \cap H_{1}^{\prime}=\varnothing$ so there is a neighborhood $W$ of $y_{2}$ in $X_{2}$ such that $W \cap \bar{U}_{2}=\varnothing$ and $\phi(W) \subset$ int $\phi\left(G^{\prime \prime} \cup U_{2}\right)$. Using the fact that $W$ and $U_{2}$ are subsets of $X_{2}$ with $W \cap U_{2}=\varnothing$ and $\phi(W) \subset$ int $\phi\left(G^{\prime \prime} \cup U_{2}\right)$ we see that $\phi\left(G^{\prime \prime} \cup W\right)=$ $\phi\left(G^{\prime \prime}\right)$. Since $y_{1} \in G^{\prime} \subset G^{\prime \prime}$ and $y_{2} \in W$ it follows that $y \in$ int $\phi\left(G^{\prime \prime} \cup W\right)$. Thus $y \in \operatorname{int}\left(G^{\prime \prime}\right) \in \mathscr{B}(n)$ and $y \in F\left(G, H_{1}\right)$ which contradicts the definition of $F\left(G, H_{1}\right)$. It follows that (a) is true and a similar argument will show (b). Hence $\mathscr{F}(n, k)$ is relatively discrete.

For each $(G, H) \in \mathcal{G}_{n} \times \mathcal{H}_{k}$ let $E(G, H)$ be an open neighborhood of $F(G, H)$ such that $E(G, H) \subset$ int $\phi\left(G^{\prime \prime} \cup H^{\prime \prime}\right)$ and $E(G, H) \cap F\left(G_{1}, H_{1}\right)=$ $\varnothing$ if $(G, H) \neq\left(G_{1}, H_{1}\right)$. Let $\mathcal{E}(n, k)=\left\{E(G, H): G \in \mathcal{G}_{n}, H \in \mathcal{K}_{n}\right\}$. To see that

$$
\{\mathscr{B}(i)\}_{i} \cup\{\mathscr{D}(j)\}_{j} \cup\{\mathcal{E}(n, k)\}_{n, k}
$$

is a quasi-development for $X$ let $U$ be an open set in $X$ and let $x \in U$. If $x_{1}$ and $x_{2}$ are the preimages of $x$ in $X_{1}$ and $X_{2}$, respectively, then there are integers $n, k$ with $G \in \mathcal{G}_{n}$ and $H \in \mathcal{H}_{k}$ such that

$$
x_{1} \in G^{\prime} \subset G^{\prime \prime} \subset \phi^{-1}(U) \text { and } x_{2} \in H^{\prime} \subset H^{\prime \prime} \subset \phi^{-1}(U) \text {. }
$$

If $x \in$ int $\phi\left(G^{\prime \prime}\right)$ then int $\phi\left(G^{\prime \prime}\right)$ is the only element of $\mathscr{B}(n)$ containing $x$ and $x \in \operatorname{St}(x, \mathscr{B}(n)) \subset U$. Similarly, if $x \in$ int $\phi\left(H^{\prime \prime}\right)$, we have $x \in \operatorname{St}(x, \mathscr{D}(k))$ $\subset U$. Otherwise, $x \in F(G, H)$ and we obtain 


$$
x \in E(G, H)=\operatorname{St}(x, \mathcal{E}(n, k)) \subset U .
$$

That concludes the proof of the theorem.

The next result, which follows very quickly as a corollary to Theorem 2.2, answers Question 2 posed by Arhangel'skii in [A].

2.3 CoRollary. Suppose $X_{1}$ and $X_{2}$ are regular developable spaces on the same set $X$. If the canonical quotient map $\phi: S \rightarrow X$ is pseudo-open, then $X$ is developable.

Proof. $X_{1}$ and $X_{2}$ are regular quasi-developable spaces so Theorem 2.2 shows that $X$ is quasi-developable. $S$ is developable, hence perfect, and it is easy to show that the property "closed sets are $G_{\delta}$ sets" is preserved under any pseudo-open finite-to-one map. Thus $X$ is a perfect quasi-developable space and therefore is developable.

The condition, in Theorem 2.2 and Corollary 2.3, that both of the spaces $X_{1}$ and $X_{2}$ be regular, is crucial as the next example shows.

2.4 ExAMPLE. There are developable spaces $X_{1}$ and $X_{2}$ with $X_{1}$ regular and $X_{2}$ Hausdorff such that $\phi: S \rightarrow X$ is pseudo-open but $X$ is not developable (or even quasi-developable).

Proof. Let $X_{1}=X_{2}=\{(x, y) \in R \times R: y \geqslant 0\}$ and let $A=\{(x, y) \in R$ $\times R: y=0\}$. Describe a topology on $X_{1}$ as follows: Points in $X_{1}-A$ are isolated and points $(a, 0) \in A$ have a neighborhood base consisting of sets of the form $\left\{(x, y) \in X_{1}: y=0,|x-a|<1 / n\right\}$, for $n \in N$. Then $X_{1}$ is a regular developable space. Describe a topology on $X_{2}$ by letting points in $X_{2}-A$ be isolated and points $(a, 0) \in A$ have a neighborhood base consisting of sets of the form

$$
\begin{aligned}
& \{(a, 0)\} \cup\left\{(x, y) \in X_{2}:|x-a|<i / n,\right. \\
& \quad \text { and } 0<y<x-a \text { or } 0<y<a-x\} .
\end{aligned}
$$

It is easy to see that $X_{2}$ is a Hausdorff developable space and the quotient map $\phi: S \rightarrow X$ is pseudo-open but the space $X$ is not developable. In fact, $X$ is semimetrizable, hence perfect and cannot be quasi-developable.

3. Other examples. We conclude with three examples. The first two, Examples 3.1 and 3.2, give negative answers to questions 3 and 4 by Arhangel'skii in [A].

3.1 EXAMPLE. There are completely regular spaces $X_{1}$ and $X_{2}$, each with a $\sigma$-disjoint base, such that $\phi: S \rightarrow X$ is pseudo-open but $X$ does not have a $\sigma$-disjoint base.

Proof. Let $X_{1}=X_{2}=\{(x, y) \in R \times R: y \geqslant 0\}$ and let $A=\{(x, y) \in R$ $\times R: y=0\}$. Describe a topology on $X_{1}$ as follows: Points in $X_{1}-A$ are isolated and points $(a, 0) \in A$ have a neighborhood base consisting of sets of the form $\left\{(x, y) \in X_{1}: y<1 / n\right.$ and $\left.y=x-a\right\}$, for $n \in N$. Describe a topology on $X_{2}$ by letting points in $X_{2}-A$ be isolated and points $(a, 0) \in A$ 
have a neighborhood base consisting of sets of the form $\left\{(x, y) \in X_{2}\right.$ : $y<1 / n$ and $y=-x+a\}$, for $n \in N$. Then $X_{1}$ and $X_{2}$ each have a $\sigma$-disjoint base (in fact, they are metrizable) and $\phi: S \rightarrow X$ is pseudo-open but the quotient space $X$ does not have a $\sigma$-disjoint base. To see this, note that $X$ is homeomorphic to the space of Example 1 of $[\mathbf{H}]$ and Heath has shown that this example is a nonscreenable Moore space, and hence could not have a $\sigma$-disjoint base.

For the next example, recall that a subset $B$ of the real numbers is called a $Q$-set if every subset of $B$ is a relative $F_{\sigma}$ set. It is known that the existence of uncountable $Q$-sets is consistent with ZFC. See [T] for further details and a discussion of the history behind $Q$-sets.

3.2 ExAMPLE (assuming the existence of an uncountable $Q$-set). There are metrizable spaces $X_{1}$ and $X_{2}$ such that $\phi: S \rightarrow X$ is pseudo-open and $X$ is normal and first countable but not metrizable.

Proof. If $B$ is an uncountable $Q$-set, let $A=B \times\{0\}$ and let $X_{1}=X_{2}=$ $A \cup\{(x, y) \in R \times R: y>0\}$. Construct topologies on $X_{1}$ and $X_{2}$ in the manner of Example 3.1. Then $X_{1}$ and $X_{2}$ are metrizable, and the quotient space $X$ is a normal metacompact nonmetrizable Moore space as shown by Heath in [H, Theorem 3].

That the quotient space $X$ in Example 3.1 (or 3.2) is metacompact is not surprising in view of a recent result by Arhangel'skiii. He solved an old problem by showing that if $f: Y \rightarrow Z$ is a pseudo-open compact mapping from a metric space $Y$ onto a space $Z$ then $Z$ is metacompact [A]. $Z$ was known to be developable, and Arhangel'skiı used the subparacompactness of $Z$ along with the collectionwise normality of $Y$ to prove that $Z$ is metacompact. In fact, with hindsight, it is relatively easy to show that if $f: Y \rightarrow Z$ is a pseudo-open compact mapping from a paracompact space $Y$ onto a subparacompact space then $Z$ is metacompact. $H$. Junnila has recently announced $[\mathbf{J}]$ the solution of a more difficult problem by showing that the pseudo-open compact image of a paracompact space is metacompact (without assuming the range is subparacompact). The next example shows that the range of such a mapping need not be subparacompact so that Arhangel'skil's approach to his problem could not be used to prove Junnila's result.

3.3 ExAmple. There are locally compact paracompact spaces $X_{1}$ and $X_{2}$ such that $\phi: S \rightarrow X$ is pseudo-open but $X$ is not subparacompact.

Proof. Let $X_{1}=X_{2}=\left[0, \omega_{2}\right) \times\left[0, \omega_{2}\right)$ and for $0<\alpha<\omega_{2}$ let

$$
H_{\alpha}=\left[0, \omega_{2}\right) \times\{\alpha\} \text { and } V_{\alpha}=\{\alpha\} \times\left[0, \omega_{2}\right) \text {. }
$$

Describe a topology on $X_{1}$ as follows: If $0<\alpha<\omega_{2}$ then neighborhoods of the point $(0, \alpha)$ must contain $(0, \alpha)$ and all but finitely many points of $H_{\alpha}$. All other points in $X_{1}$ are isolated. Describe a topology on $X_{2}$ as follows: If $0<\alpha<\omega_{2}$ then neighborhoods of $(\alpha, 0)$ must contain $(\alpha, 0)$ and all but finitely many points of $V_{\alpha}$. All other points in $X_{2}$ are isolated. It follows that 
$X_{1}$ and $X_{2}$ are locally compact paracompact spaces and $\phi: S \rightarrow X$ is pseudoopen. The image space $X$ is the same as the space in Example 4.1 in [Bu] and it is shown there that $X$ is not subparacompact.

\section{REFERENCES}

[A] A. V. Arhangel'skil, The intersection of topologies, and pseudo-open compact mappings, Soviet Math. Dokl. 17 (1976), 160-163.

[Be] H. R. Bennett, On quasi-developable spaces, General Topology and Appl. 1 (1971), 253-262.

[BL] H. R. Bennett and D. J. Lutzer, $A$ note on weak $\theta$-refinability, General Topology and Appl. 2 (1972), 49-54.

[Bu] D. K. Burke, On subparacompact spaces, Proc. Amer. Math. Soc. 23 (1969), 655-663.

[H] R. Heath, Screenability, pointwise paracompactness and metrization of Moore spaces, Canad. J. Math. 16 (1964), 763-770.

[J] H. Junnila, On pseudo-open mappings of paracompact spaces, Notices Amer. Math. Soc. 25 (1978), p. A-138, Abstract 752-54-16.

[T] F. D. Tall, Set-theoretic consistency results and topological theorems concerning the normal Moore space conjecture and related problems, Thesis, Univ. of Wisconsin, Madison, 1969; Dissertationes Math. 148 (1977), 1-53.

Department of Mathematics, Miami University, Oxford, OhIo 45056 